\title{
Postoperative Fractionated Stereotactic Radiosurgery to the Tumor Bed for Surgically Resected Brain Metastases
}

Ryan K. Cleary ${ }^{1}$, Jessica Meshman ${ }^{1}$, Michael Dewan ${ }^{2}$, Liping Du ${ }^{3}$, Anthony J. Cmelak ${ }^{1}$, Guozhen Luo $^{1}$, Manuel Morales-Paliza ${ }^{1}$, Kyle Weaver ${ }^{2}$, Reid Thompson ${ }^{2}$, Lola B. Chambless 2 , Albert Attia ${ }^{1}$

1. Department of Radiation Oncology, Vanderbilt University Medical Center 2. Department of Neurological Surgery, Vanderbilt University Medical Center 3. Center for Quantitative Sciences, Vanderbilt University School of Medicine

$\square$ Corresponding author: Ryan K. Cleary, ryan.k.cleary@vanderbilt.edu Disclosures can be found in Additional Information at the end of the article

\section{Abstract}

Introduction

Stereotactic radiosurgery (SRS) is increasingly used as an alternative to whole brain radiotherapy (WBRT) following surgical resection of brain metastases. We analyzed the outcomes of postoperative frameless fractionated stereotactic radiosurgery (fSRS) cases for surgically resected brain metastases at our institution.

Materials and Methods

We performed a retrospective review of 85 patients who underwent fSRS to 87 resection beds from 2006 - 2014 with a median follow-up of 6.4 months. Clinically relevant outcomes were assessed with analysis to determine predictors of these outcomes.

Results

The median target volume was $9.8 \mathrm{~cm}^{3}\left(1.1-43.1 \mathrm{~cm}^{3}\right)$. The most frequently used fractionation scheme was 3,000 cGy in five fractions. The rates of local control (LC), distant brain failure (DBF), and overall survival (OS) at one-year were 87\%, 52\%, and 52\%, respectively. Five patients (5.9\%) experienced Grade $>2$ toxicity related to fSRS, including seizures (two), symptomatic radionecrosis (two), and potential treatment-related death (one). A multivariable analysis revealed that tumor volume $(\mathrm{p}<0.001)$ and number of fractions $(\mathrm{p}<0.001)$ were

Received 05/04/2017

Review began 05/11/2017 Review ended 05/23/2017 Published 05/26/2017

CC Copyright 2017

Cleary et al. This is an open access article distributed under the terms of the Creative Commons Attribution License CC-BY 3.0., which permits unrestricted use, distribution, and reproduction in any medium, provided the original author and source are credited. associated with LC, while recursive partitioning analysis (RPA) class $(p<.0001)$, tumor volume $(p=.0181)$, and the number of fractions $(p=.0181)$ were associated with OS.

\section{Conclusions}

Postoperative fSRS for surgically resected brain metastases is well-tolerated and achieves durable LC. Further studies are needed to determine the optimal dose and fractionation for fSRS as well as to compare outcomes with WBRT.

Categories: Radiation Oncology, Neurosurgery, Oncology
How to cite this article

Cleary R K, Meshman J, Dewan M, et al. (May 26, 2017) Postoperative Fractionated Stereotactic Radiosurgery to the Tumor Bed for Surgically Resected Brain Metastases. Cureus 9(5): e1279. DOI 10.7759/cureus. 1279 
Keywords: stereotactic radiosurgery, brain metastases, fractionated radiotherapy, frameless stereotactic radiosurgery, fsrs

\section{Introduction}

Whole-brain radiation therapy (WBRT) became the standard of care following surgical resection of a single brain metastasis based on the results published by Patchell, et al. [1]. Their randomized trial showed decreased local and distant brain failure (DBF) rates after WBRT versus observation alone. Reduced incidence of neurological death was also seen with WBRT, though an overall survival (OS) benefit was not shown. Focal techniques, such as stereotactic radiosurgery (SRS), emerged as an alternative to WBRT in order to provide local control (LC) at the resection cavity, while sparing radiation to normal brain. Results from multiple series show LC on the order of $75-85 \%$ with DBF rates of approximately $40-60 \%$ at one year [2-3].

Fractionated stereotactic radiosurgery (fSRS) has been employed in treating surgical cavities of increasing size in an attempt to maintain LC while reducing the risk of treatment toxicity [4-7]. Herein, we report on the postoperative fSRS experience at Vanderbilt University Medical Center.

\section{Materials And Methods}

Between February 2006 and November 2014, 85 patients underwent surgical resection of a brain metastasis followed by fSRS. Two of these patients developed an additional brain metastasis at a separate site in the brain requiring surgical resection followed by adjuvant stereotactic radiation for a total of 87 treated resection cavities. Additional contemporaneous metastases discovered on imaging were treated with SRS according to the usual fashion.

Data regarding treatment of these patients were acquired retrospectively. Prior to the acquisition of data, Vanderbilt University Medical Center Institutional Review Board approval (\#150276) was obtained.

\section{Radiosurgery technique}

Radiosurgery was performed with a Novalis $\mathrm{Tx}^{\mathrm{TM}}$ linear accelerator-based radiosurgery platform (Varian Medical Systems, Palo Alto, CA). ExacTrac ${ }^{\circledR}$ (BrainLab, Munich, Germany) stereoscopic $\mathrm{kV}$ x-ray monitoring for patient localization was employed for all cases starting in 2009. Using this frameless method, patients were immobilized supine on the treatment table with a custom thermoplastic SRS mask. All patients underwent postoperative gadoliniumenhanced magnetic resonance imaging (MRI) of the brain within one to two days following surgery to assess the extent of the surgical resection. An additional 1-mm slice thickness contrasted MRI of the brain was performed within one to three weeks of radiosurgery for treatment planning purposes. Typically, fSRS was started within four weeks following the date of surgery.

The edge of the resection cavity with any associated residual contrast enhancement was designated the tumor volume. Contouring was performed by the radiation oncologist and the operating neurosurgeon. Use of an expansion margin of 1-2 mm was performed at the discretion of the treating radiation oncologist. The prescription dose and fractionation were determined by the radiation oncologist with consideration for cavity size and surrounding normal tissue constraints.

\section{Follow-up}

Patients received regular follow-up with the treating radiation oncologist every two to three months for the first year, then every three to six months thereafter. Neurological examination 


\section{Cureus}

was performed at each visit. The Radiation Therapy Oncology Group (RTOG) grading system was used to assess toxicity. Treatment-related death was defined as death within 30 days of completion of radiosurgery. Determination of radionecrosis of the brain was made on MRI by the multidisciplinary neuro-oncology tumor board, which included the treating radiation oncologist, neurosurgeon, pathologist, and radiologist. Neurologic death was defined as intracranial progression at the time of death in the absence of systemic disease progression [1].

\section{Statistical analysis}

Patient demographics and disease and treatment characteristics were summarized using frequencies and relative frequencies for categorical variables. Medians and ranges were used for continuous variables. Times to death, local recurrence (LR), and DBF were calculated from the date of radiosurgery to the date of the defined event. For OS, the event was death and those who were alive at the last follow-up were censored. For LC (defined as the absence of radiographic evidence of disease recurrence within $1 \mathrm{~cm}$ of the surgical resection cavity), the event of interest was LR, while death without LR was considered as a competing risk; those with neither LR nor death were censored at the last follow-up. For distant control (defined as lack of development of new brain metastases or leptomeningeal disease outside of the treatment volume), the event of interest was DBF, and death without DBF was as a competing risk; those with neither DBF nor death were censored at the last follow-up. For OS, the data for the 85 patients who were considered independent were used. The Kaplan-Meier method was used to estimate the survival rates, and multivariable Cox regression with robust standard errors was performed to estimate the adjusted hazard ratios (HR). For LC and distant control, competing risk survival analysis using Fine and Gray's method [8-9] was performed using the data for the 87 treated resection cavities that were considered independent. All the multivariable regression models were pre-specified to increase the precision of estimation and avoid potential confounding. Covariates, such as age, recursive partitioning analysis (RPA) class, primary tumor site, tumor volume, and the number of fractions (2, 3, and 4-5) were included in the Cox regression model for OS. Age, sex, RPA class, primary tumor site, tumor volume, and the number of fractions (2, 3, and 4-5) were included in the local control multivariable model. In addition to these covariates, margin use or not, the number of tumors in the brain and systemic disease status were included in the model for distant control. The two-sided nominal level of 0.05 was considered as statistically significant for all the tests. All statistical analyses were performed using software R version 3. 2.4, including packages “ survival”, "Hmisc", “rms”, and “mstate”.

\section{Results}

At our institution, 85 patients underwent postoperative fSRS following surgical resection of a brain metastasis between February 2006 and November 2014. Two patients underwent resection of an additional brain metastasis at a different time point for a total of 87 resection cavities. Baseline patient characteristics are listed in Table 1. The median age of our patients was 58.9 years (range: 30.9 - 85.3). Over half of our patients were RPA class 2 (52.9\%). Nonsmall cell lung cancer (NSCLC) (40.0\%) and melanoma (21.2\%) were the most common primary histologic subtypes treated. Sixty-two patients (72.9\%) had a single resected brain metastasis, whereas the remaining patients had additional brain metastases managed with SRS. Four patients (4.7\%) had received prior WBRT.

\section{Variable}

No. of patients

Age (median in y)

\section{Patients}

85

58.9 


\section{Cureus}

Sex

Male

$52(61.2 \%)$

Female

$33(38.8 \%)$

RPA Class

1

$24(28.2 \%)$

2

$45(52.9 \%)$

3

$16(18.8 \%)$

Primary

Breast

$8(9.4 \%)$

Colorectal

$3(3.5 \%)$

Head and neck

$1(1.2 \%)$

Melanoma

$18(21.2 \%)$

NSCLC

$34(40.0 \%)$

Renal cell

$9(10.6 \%)$

SCLC

$2(2.3 \%)$

Other

$10(11.8 \%)$

Number of brain metastases

1

$62(72.9 \%)$

2

$9(10.6 \%)$

3

$6(7.1 \%)$

$4+$

$8(9.4 \%)$

Systemic disease burden

None

$36(42.3 \%)$

Oligometastatic (

$18(21.2 \%)$

Widespread

$31(36.4 \%)$

Systemic disease status

Stable

$43(50.6 \%)$

Progressive

$40(47.1 \%)$

Unknown

$2(2.4 \%)$

Prior WBRT

Yes

$4(4.7 \%)$ 


\section{Cureus}

\section{TABLE 1: Patient Characteristics}

RPA: recursive partitioning analysis; NSCLC: non-small cell lung cancer; SCLC: small cell lung cancer; WBRT: whole brain radiotherapy. Values are number (percentage) unless otherwise noted.

All resection cavities received radiosurgery delivered in two to five fractions (See Table 2 for treatment characteristics). The median size of the resection cavities in our series was $9.8 \mathrm{cc}$ (range: 1.1 - $43.1 \mathrm{cc}$ ). The median prescription dose was 3,000 cGy (range: 1,800 - 3,500).

\section{Parameter}

Tumor volume $\left(\mathrm{cm}^{3}\right)$

Fractions

2

3

5

Prescribed dose (cGy)

Maximum dose (cGy)

Margin use

Yes

No

\section{Value}

$9.8(1.1-43.1)^{*}$

$17(19.5 \%)$

$27(31.0 \%)$

$5(5.6 \%)$

$38(43.7 \%)$

$3,000(1,800-3,500)$

$3,549(2,134-4,388)$

$36(41.3 \%)$

$51(58.7 \%)$

\section{TABLE 2: Treatment Parameters}

cGy - centigray

Values are median (range) or number (percentage).

*There is one missing datum, making 86 known tumor volumes.

The median follow-up time for our cohort was 6.4 months (range: 0.6 - 93.5). The estimated six-month and one-year cumulative incidence rates for LR in the presence of competing risk were $10 \%$ and $13 \%$, respectively (Figure 1). Statistically significant predictors of LC from the multivariable competing risk survival analysis were tumor volume $(\mathrm{p}=<.0001)$ and number of fractions $(\mathrm{p}=<.0001)$. The analysis also revealed a statistically significant effect modification of tumor volumes on the number of fractions; the effect of increasing number of fractions on 


\section{Cureus}

improving local control was most important with larger tumor volumes.

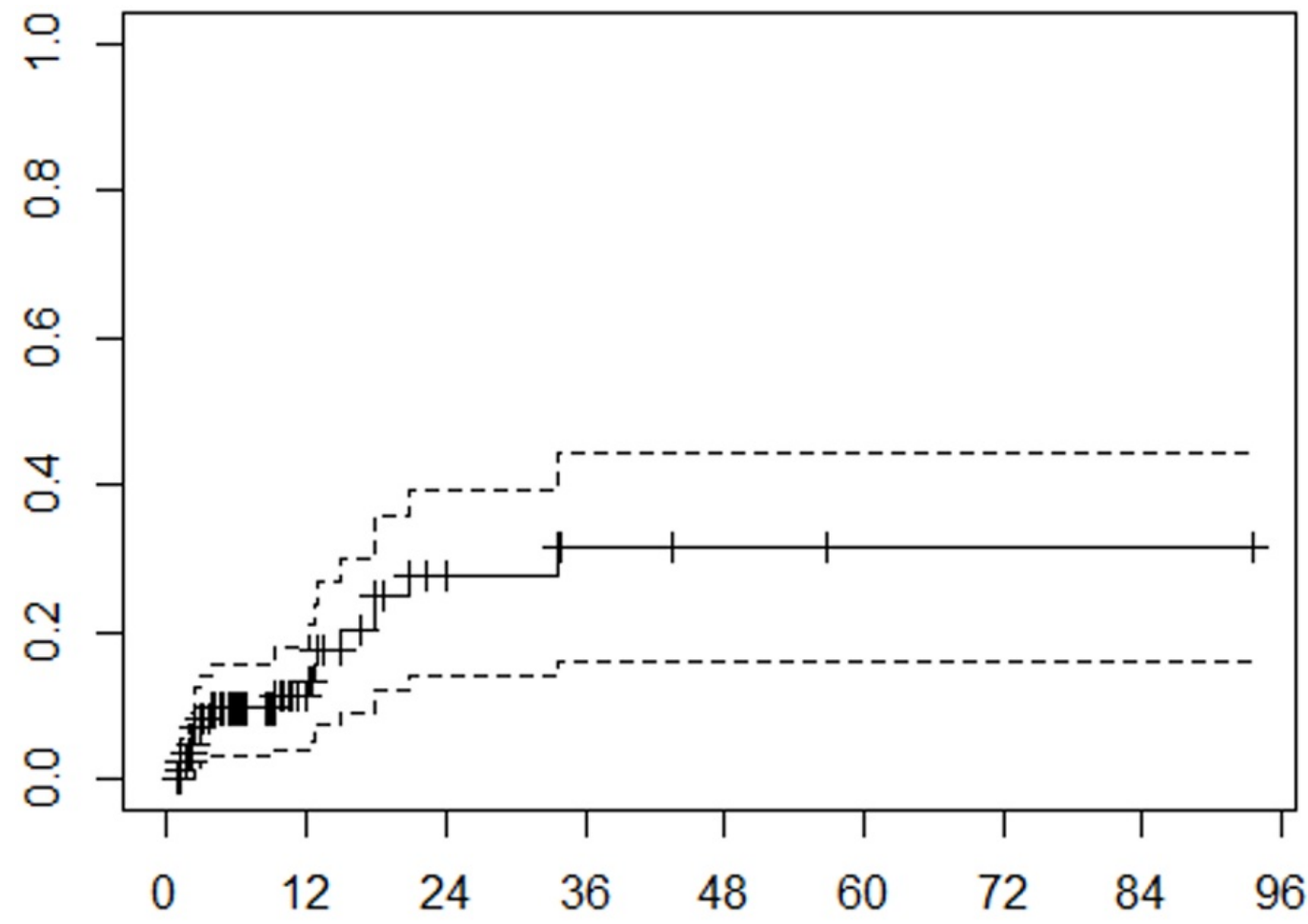

\section{FIGURE 1: Local recurrence}

Cumulative incidence function of local recurrence. Months from treatment on x-axis, cumulative incidence on y-axis. Tick marks on the solid curve represent censored data. Dashed lines represent $95 \%$ confidence interval.

The estimated cumulative incidence rates for DBF at six months and one year were $41 \%$ and $52 \%$, respectively (Figure 2). Ten patients experienced leptomeningeal failure while the remainder had new brain metastases. Multivariable analysis revealed that tumor volume $(\mathrm{p}=$ .0222) and systemic disease status $(\mathrm{p}=.0161)$ were statistically significantly associated with DBF. 


\section{Cureus}

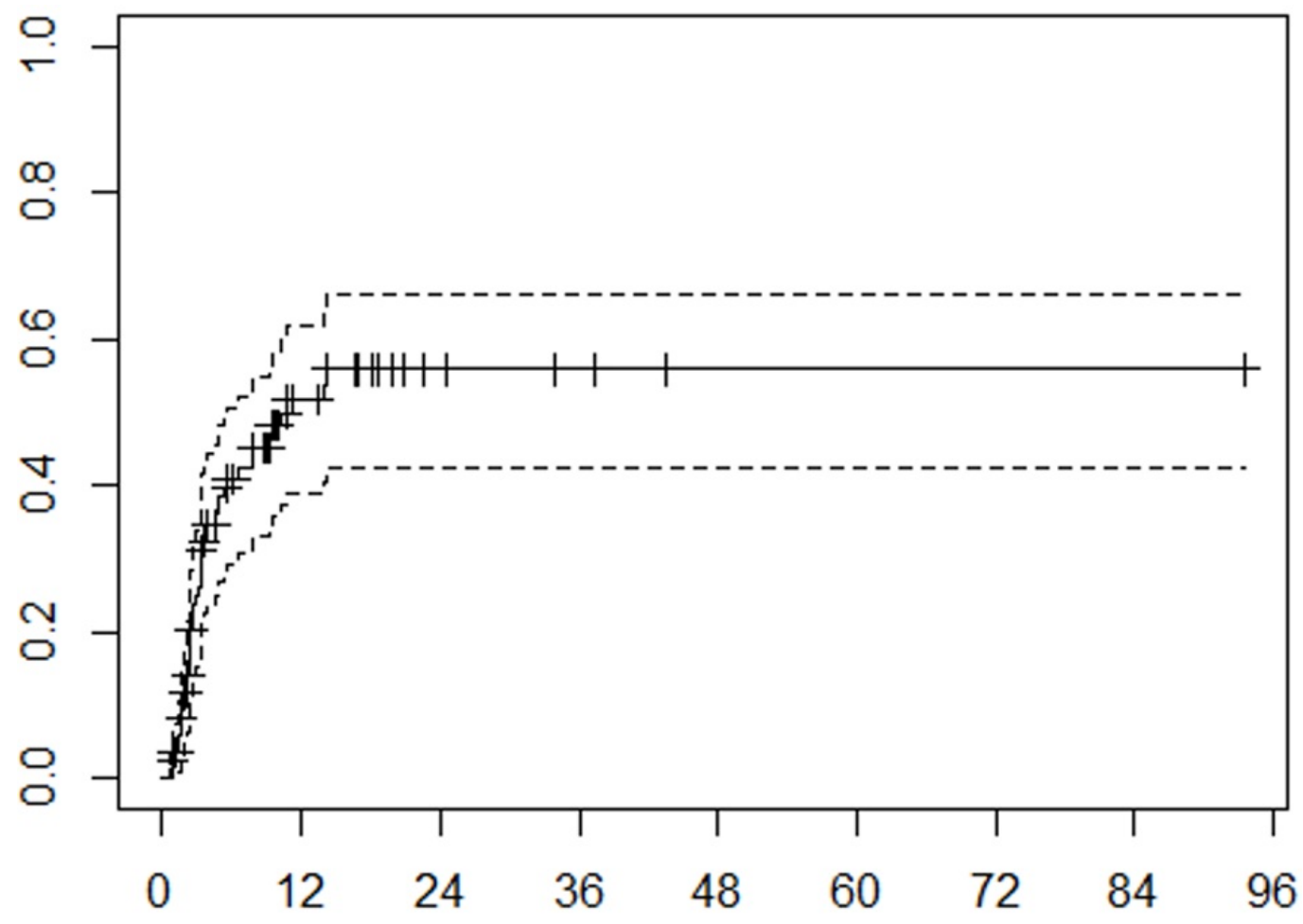

\section{FIGURE 2: Distant brain failure}

Cumulative incidence function of distant brain failure. Months from treatment on x-axis, cumulative incidence on $y$-axis. Tick marks on the solid curve represent censored data. Dashed lines represent $95 \%$ confidence interval.

The median OS for our patients was 13.0 months. The estimated actuarial OS rates at six months and one year were $68 \%$ and $52 \%$, respectively (Figure 3$)$. RPA class $(\mathrm{p}<.0001)$, tumor volume $(\mathrm{p}=.0181)$, and number of fractions $(\mathrm{p}=.0181)$ were statistically significantly associated with OS on multivariable analysis. 


\section{Cureus}

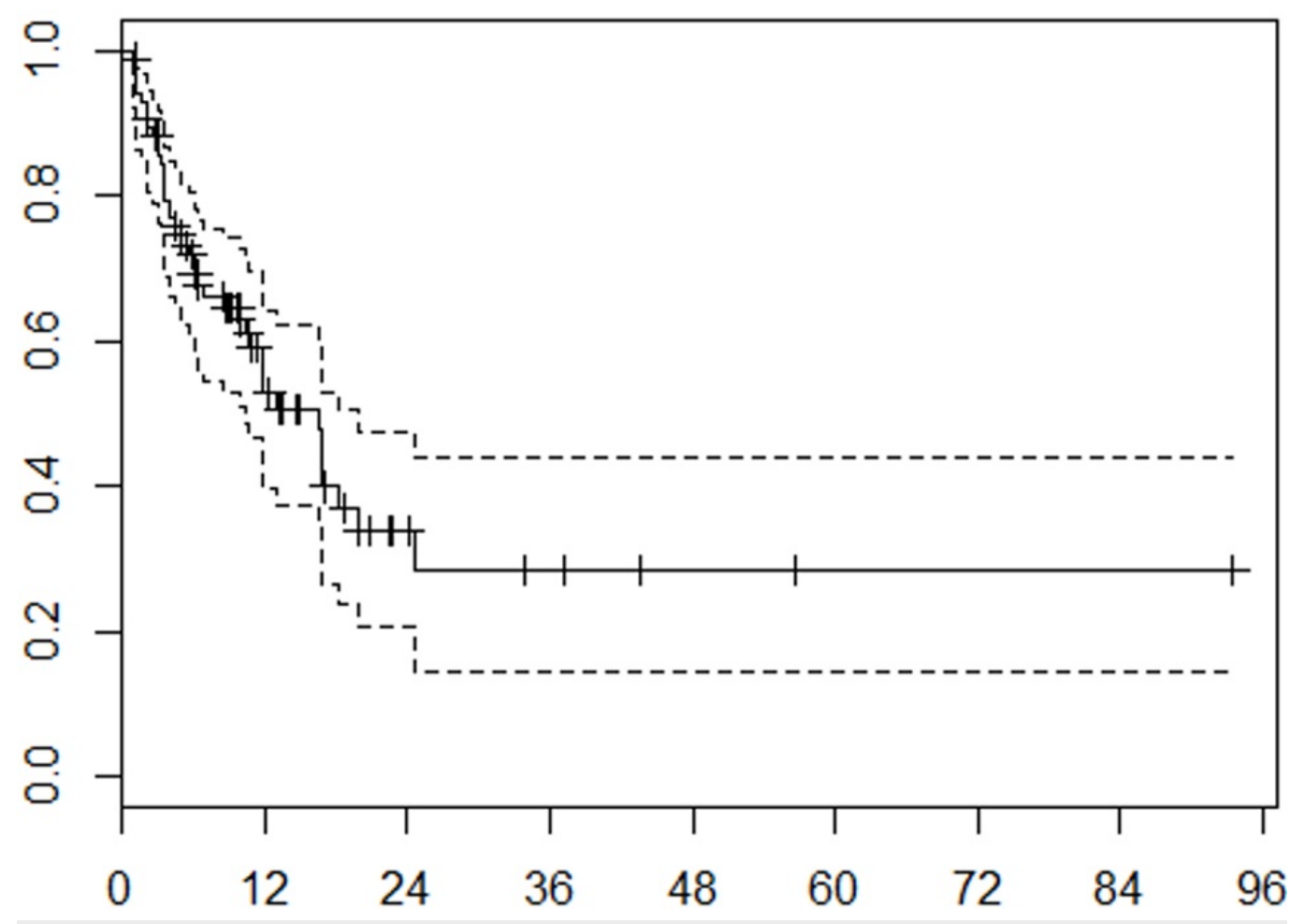

FIGURE 3: Overall survival

Kaplan-Meier analysis of overall survival. Months from treatment on $\mathrm{x}$-axis, probability on y-axis. Tick marks on the solid curve represent censored data. Dashed lines represent $95 \%$ confidence interval.

Five patients (5.9\%) experienced Grade > 2 toxicity related to fSRS. One patient died within 30 days of receiving fSRS of 3,000 cGy in five fractions to a $5.6 \mathrm{cc}$ cerebellar resection cavity. His course was complicated by vasogenic edema and disease progression causing obstructive hydrocephalus which eventually led to his demise. Another two patients experienced seizures shortly after undergoing radiosurgery (2.4\%). Symptomatic radionecrosis was seen in two patients over the course of follow-up (2.4\%). Neurologic death was seen in 16 patients (18.8\%).

\section{Discussion}

Whole brain radiotherapy has been shown to reduce the rates of LR and DBF following surgical resection of a single brain metastasis [1]. Patients in the Patchell trial who underwent WBRT were less likely to experience LR ( $10 \%$ vs. $46 \%$, respectively), DBF (14\% vs. $37 \%$ ), or recurrence anywhere in the brain (18\% vs. $70 \%$ ) versus observation. However, no OS benefit was seen with the addition of WBRT. Moreover, WBRT has been associated with neurocognitive impairment [10]. Therefore, patients are increasingly being managed in the postoperative setting with radiosurgery alone to the surgical resection cavity and any remaining sites of intracranial metastatic disease. Jensen, et al. showed that high-resolution MRI and cavity-directed radiosurgery could be used following resection of a brain metastasis to spare over $50 \%$ of patients from undergoing WBRT at one year [11].

Traditionally, patients have been treated with single fraction SRS with recent series showing local control at one year from 73-94\% [3, 12-14]. A prospective, randomized trial of singlefraction SRS to the surgical cavity versus observation alone after brain metastasis resection was performed at the MD Anderson Cancer Center (MDACC) and recently presented at the American Society of Radiation Oncology (ASTRO) 2016 Annual Meeting [15]. This trial showed improved 
one-year LC for postoperative SRS as compared to observation alone ( $72 \%$ vs. $45 \%$, respectively), although survival was the same for both groups. Many centers are moving toward fSRS for larger resection cavities, given the concern for normal tissue toxicity. Our retrospective study of patients treated with fSRS following surgical resection of a brain metastasis is one of the larger series in the literature and shows excellent local control (87\%) at one year. This is in line with several other published series of fSRS which report one-year local control of 79-93\%. [2, 4, 6-7]. Our median cavity size was similar to these series at $9.8 \mathrm{cc}$, and cavities up to $43 \mathrm{cc}$ in size were treated safely. Distant brain failure was $48 \%$ at one year, which is also comparable to these series. Although one patient died shortly after fSRS as a possible complication of treatment versus disease progression, the treatment was generally well-tolerated with few side effects, including only two cases of symptomatic radionecrosis.

The most intriguing finding from our study was the association between the increasing number of fractions and improved local tumor control. This effect appears to be most pronounced with larger tumor volumes. One explanation for this finding is that through increasing the number of fractions, a larger biologically effective dose (BED) was delivered to the cavity as compared to administering a smaller number of fractions due to physician concern for long-term toxicity with larger fraction sizes and/or fewer fractions, especially as cavity size increases. Previous single fraction SRS series for intact brain metastases have shown that local control decreases with increasing tumor volume [16-17]. This has been noted in series of brain metastases treated with multimodality therapy, including fSRS [7]. Notably, the incidence of radionecrosis after single-fraction SRS has been shown to increase significantly when the volume receiving $12 \mathrm{~Gy}$ is $>8 \mathrm{~cm}^{2}[18]$. Fractionated stereotactic radiosurgery offers the ability to increase the dose to the cavity while minimizing side effects as the rate of symptomatic radionecrosis was only $2.4 \%$ in the current series. The most frequently used fractionation scheme for our patients was 600 cGy $x$ five fractions delivered on consecutive days. The $\mathrm{BED}_{10}$ for this scheme is roughly equivalent to $17.5 \mathrm{~Gy}$ delivered in a single fraction. As a point of reference, the recently completed Phase III Randomized Study of Post-Surgical Stereotactic Radiosurgery Versus Whole-Brain Radiotherapy in Patients with Resected Metastatic Brain Metastases (NCCTGN107C) trial comparing post-surgical SRS with WBRT for resected metastatic brain disease dictated single-fraction prescription doses from 12-20 Gy depending on cavity size [19]. Although the initial results from this trial presented at ASTRO 2016 showed similar OS and improved cognitive outcomes with postoperative SRS versus WBRT, the surgical bed control rate in the SRS arm was significantly worse at 12 months as compared to WBRT (55.6\% vs $78.2 \%$, respectively). The numerically higher incidence of surgical bed failure in the singlefraction SRS arm of NCCTG-N107C and in the prospective MDACC trial, as compared to the current series, suggests that further studies are needed to determine if fSRS may provide the means to maintain local control while minimizing side effects, especially with larger cavity sizes.

The limitations of our study include the short median follow-up time, as well as the number of patients examined. Both of these limit the power of our study to determine predictors of patient outcome via multivariable analysis. Additionally, our study is limited by its retrospective nature without a control group treated with alternate modalities. However, our study provides further evidence regarding the efficacy and safety of postoperative fSRS for the treatment of brain metastases.

\section{Conclusions}

Stereotactic radiosurgery is an emerging alternative to WBRT following surgical resection of brain metastases. We performed a retrospective review of patients treated at our institution with frameless fSRS after undergoing neurosurgical extirpation of an intracranial metastasis. Results from our study show that fSRS to the postoperative resection cavity with a median dose of $30 \mathrm{~Gy}$ in five fractions provides excellent LC ( $87 \%$ at one year) with minimal neurotoxicity, 
thus sparing many patients the untoward neurocognitive effects of WBRT. Additional multivariate analysis revealed that tumor volume and number of fractions are associated with LC, suggesting that fractionation may play a role in improving LC rates in these patients. Further studies are needed determine the optimal dose and fractionation for multimodality treatment of brain metastases with fSRS.

\section{Additional Information \\ Disclosures}

Human subjects: Consent was obtained by all participants in this study. Vanderbilt University Medical Center IRB issued approval 150276. Animal subjects: All authors have confirmed that this study did not involve animal subjects or tissue. Conflicts of interest: In compliance with the ICMJE uniform disclosure form, all authors declare the following: Payment/services info: All authors have declared that no financial support was received from any organization for the submitted work. Financial relationships: Albert Attia declare(s) personal fees from Brainlab. Albert Attia declare(s) personal fees from Qfix. Albert Attia declare(s) personal fees from AstraZeneca. Other relationships: All authors have declared that there are no other relationships or activities that could appear to have influenced the submitted work.

\section{References}

1. Patchell RA, Tibbs PA, Regine WF, et al.: Postoperative radiotherapy in the treatment of single metastases to the brain: a randomized trial. JAMA. 1998, 280:1485-89. 10.1001/jama.280.17.1485

2. Soltys SG, Adler JR, Lipani JD, et al.: Stereotactic radiosurgery of the postoperative resection cavity for brain metastases. Int J Radiat Oncol. 2008, 70:187-93. 10.1016/j.ijrobp.2007.06.068

3. Quigley MR, Fuhrer R, Karlovits S, et al.: Single session stereotactic radiosurgery boost to the post-operative site in lieu of whole brain radiation in metastatic brain disease. J Neurooncol. 2008, 87:327-32. 10.1007/s11060-007-9515-Z

4. Minniti G, Esposito V, Clarke E, et al.: Multidose stereotactic radiosurgery $(9 \mathrm{~Gy} \times 3)$ of the postoperative resection cavity for treatment of large brain metastases. Int J Radiat Oncol. 2013, 86:623-29. 10.1016/j.ijrobp.2013.03.037

5. Ling DC, Vargo JA, Wegner RE, et al.: Postoperative stereotactic radiosurgery to the resection cavity for large brain metastases: clinical outcomes, predictors of intracranial failure, and implications for optimal patient selection. Neurosurgery. 2015, 76:150-57. 10.1227/NEU.0000000000000584

6. Kelly PJ, Lin YB, Yu AY, et al.: Stereotactic irradiation of the postoperative resection cavity for brain metastasis: a frameless linear accelerator-based case series and review of the technique. Int J Radiat Oncol Biol Phys. 2012, 82:95-101. 10.1016/j.ijrobp.2010.10.043

7. Ahmed KA, Freilich JM, Abuodeh Y, et al.: Fractionated stereotactic radiotherapy to the postoperative cavity for radioresistant and radiosensitive brain metastases. J Neurooncol. 2014, 118:179-86. 10.1007/s11060-014-1417-2

8. Geskus RB: Cause-specific cumulative incidence estimation and the fine and gray model under both left truncation and right censoring. Biometrics. 2011, 67:39-49. 10.1111/j.15410420.2010.01420.x

9. Fine JP, Gray RJ: A proportional hazards model for the subdistribution of a competing risk . J Am Stat Assoc. 1999, 94:496-509. 10.2307/2670170

10. Chang EL, Wefel JS, Hess KR, et al.: Neurocognition in patients with brain metastases treated with radiosurgery or radiosurgery plus whole-brain irradiation: a randomised controlled trial. Lancet Oncol. 2009, 10:1037-44. 10.1016/\$1470-2045(09)70263-3

11. Jensen CA, Chan MD, McCoy TP, et al.: Cavity-directed radiosurgery as adjuvant therapy after resection of a brain metastasis. J Neurosurg. 2011, 114:1585-91. 10.3171/2010.11.JNS10939

12. Jagannathan J, Yen CP, Ray DK, et al.: Gamma Knife radiosurgery to the surgical cavity following resection of brain metastases. J Neurosurg. 2009, 111:431-38. 10.3171/2008.11.JNS08818

13. Ojerholm E, Lee JY, Thawani JP, et al.: Stereotactic radiosurgery to the resection bed for 
intracranial metastases and risk of leptomeningeal carcinomatosis. J Neurosurg. 2014, $121: 75-83$.

14. Iorio-Morin C, Masson-Côté L, Ezahr Y, et al.: Early Gamma Knife stereotactic radiosurgery to the tumor bed of resected brain metastasis for improved local control. J Neurosurg. 2014, 121:69-74.

15. Mahajan A, Ahmed S, Li J, et al.: Postoperative stereotactic radiosurgery versus observation for completely resected brain metastases: results of a prospective randomized study. Int J Radiat Oncol Biol Phys. 2016, 96:S2. 10.1016/j.ijrobp.2016.06.022

16. Shaw E, Scott C, Souhami L, et al.: Single dose radiosurgical treatment of recurrent previously irradiated primary brain tumors and brain metastases: final report of RTOG protocol 90-05. Int J Radiat Oncol Biol Phys. 2000, 47:291-98. 10.1016/S0360-3016(99)00507-6

17. Han JH, Kim DG, Chung HT, et al.: Radiosurgery for large brain metastases. Int J Radiat Oncol Biol Phys. 2012, 83:113-20. 10.1016/j.ijrobp.2011.06.1965

18. Blonigen BJ, Steinmetz RD, Levin L, et al.: Irradiated volume as a predictor of brain radionecrosis after linear accelerator stereotactic radiosurgery. Int J Radiat Oncol Biol Phys. 2010, 77:996-1001. 10.1016/j.ijrobp.2009.06.006

19. Brown PD, Ballman KV, Cerhan J, et al.: N107C/CEC.3: A phase III trial of post-operative stereotactic radiosurgery (SRS) compared with whole brain radiotherapy (WBRT) for resected metastatic brain disease. Int J Radiat Oncol Biol Phys. 2016, 96:937.

10.1016/j.ijrobp.2016.09.045 